\title{
Venous Thromboembolism Among Military Combat Casualties
}

\author{
Raymond Fang ${ }^{1}$ - Carlos J. Rodriguez ${ }^{2}$
}

Published online: 19 February 2016

(C) Springer International Publishing AG (outside the USA) 2016

\begin{abstract}
Venous thromboembolism (VTE) complicates trauma no matter whether injuries occur in the civilian or combat setting. Combat casualty care is further challenged by the requirement to rapidly evacuate patients by air from the combat zone to definitive care destinations in the USA. The Joint Trauma System (JTS) is charged with ensuring optimal management of the combat injured across the global care continuum. The JTS first released guidelines for "Prevention of deep venous thrombosis" in December 2004 and regularly revises it to ensure it reflects current, best practice. This review presents a perspective on VTE impact on wartime care and the US military medical system's effort to prevent these complications.
\end{abstract}

Keywords Military medicine - Venous thromboembolism . Deep vein thrombosis $\cdot$ Pulmonary embolism $\cdot$ Risk factors . Combat air travel

This article is part of the Topical Collection on The Military Perspective

The opinions presented are the authors own, and do not reflect the official position of the US Air Force, US Navy, Department of Defense, or the US Government.

Raymond Fang

rfang@umm.edu

Carlos J. Rodriguez

carlos.j.rodriguez4.mil@mail.mil

1 United States Air Force Center for the Sustainment of Trauma and Readiness Skills, R Adams Cowley Shock Trauma Center, University of Maryland Medical Center, 22 South Greene Street, Baltimore, MD 21201, USA

2 Walter Reed National Military Medical Center, 8901 Wisconsin Ave, Bethesda, MD 20902, USA

\section{Introduction}

Venous thromboembolism (VTE) complicates trauma no matter whether injuries occur in the civilian or combat setting. In civilian trauma, reported VTE rates range from $0.36-58 \%$ depending on (1) the population studied, (2) the VTE prophylaxis measures taken, and (3) the diagnostic protocols utilized $[1,2,3 \cdot]$. Reported combat trauma VTE rates range from $2.3 \%$ to over $23 \%[4,5 \cdot]$, and have been noted to be particularly high among the severe multi-system injuries that have characterized the past decade - plus of combat operations in Iraq and Afghanistan. This review presents a perspective on the challenges of VTE for combat casualty care and the US military medical system's effort to prevent post-traumatic VTE complications among wounded service members.

\section{Civilian Versus Combat Post-Trauma Venous Thromboembolism Rates}

In 2011, Knudson et al. reported on a "contemporary" cohort of civilian trauma patients abstracted from Level 1 and 2 trauma center admissions from 2007-2009 reported to the American College of Surgeons National Trauma Data Bank (NTDB) [2]. Ninety percent of the patients in this analysis were injured by blunt mechanisms reflective of typical civilian mechanism of injury. The mean Injury Severity Score (ISS) was $11 ; 24 \%$ of the patients sustained an ISS greater than 15 . In these 888,652 patients, the rate of deep venous thrombosis (DVT) was $1.06 \%$ and pulmonary embolus (PE) was $0.42 \%$. In comparison, civilian studies reporting VTE rates in specific, high-risk trauma groups report much higher rates. For example, VTE rates for civilian patients with pelvic and acetabular fractures approach $12 \%[3 \cdot, 6,7]$. 
In 2014, Hutchison et al. retrospectively analyzed over 26, 000 combat trauma casualties abstracted from the Joint Theater Trauma Registry (now renamed the Department of Defense Trauma Registry (DoDTR)) for the period between October 2001 to July 2011 [4]. The overall VTE rate was $2.2 \%$ with a $1.0 \%$ DVT rate and a $0.8 \%$ PE rate. Ninety-four $(0.4 \%)$ patients were diagnosed with both DVT and PE. In assessing risk factors, the authors identified amputations to be associated with a significantly increased risk. Their subgroup analysis of casualties sustaining amputations found a very substantial VTE rate of $17.3 \%$.

Two institution-specific analyses of combat-related VTE rates at role 5 (US) medical treatment facilities have been published:

In 2011, Gillern et al. utilized retrospective data from the National Naval Medical Center (NNMC) [Bethesda, Maryland] Combat Trauma Registry to specifically evaluate casualties with wartime extremity injuries and included patients who (1) underwent at least one orthopedic procedure performed upon arrival to the USA or (2) sustained amputation before arrival [8]. In this group of 263 patients treated from March 2003-December 2007, 15 patients were diagnosed with DVT (5.7\%) and 15 with PE (5.7\%). Three patients were diagnosed with both DVT and PE and were included in both groups so the total VTE rate was $10.3 \%$. Of note, one death was attributed to an acute PE.

In 2013, Caruso et al. reported a single institution rate of $11.4 \%$ for new VTE occurring after arrival to the USA between November 2010 to November 2012 at the Walter Reed National Military Medical Center [Bethesda, Maryland] [5•]. During the 2-year study period, 565 combat casualties were admitted with 484 patients eligible for evaluation per the study protocol. Sixty-six patients arrived on therapeutic anticoagulation for VTE diagnosed prior to arrival and were excluded. Fifty-five casualties (11.4 \%) were diagnosed with a VTE event during their hospitalization, 35 (63\%) PEs, 13 (24\%) upper extremity DVTs, and 8 (13\%) lower extremity DVTs. There were no deaths due to PE in the study cohort. The total VTE rate was $21.4 \%(121 / 565)$ when the 66 patient already treated on arrival are added to the cohort.

\section{Risk Factors for the Development of Venous Thromboembolism}

Virchow's Triad of endothelial injury, hypercoagulability, and stasis are well-known risk factors for VTE complications in trauma patients. Multiple authors have sought to define additional risk factors for the development of post-traumatic VTE. Geerts et al. first prospectively identified in 1994 five independent risk factors for post-traumatic VTE following major trauma which remain valid: older age, blood transfusion, surgery, femur and tibia fractures, and spinal cord injury [1]. Knudson et al. identified similar risk factors: older age, greater than three ventilator days, injuries to the chest, lower extremities or spine, pelvic fractures, and shock [2]. In 2012, Rogers et al. evaluated over 16,600 trauma patients at Lancanster General Hospital (Lancaster, Pennsylvania) to develop a VTE risk factor model [9]. They validated their model by applying it against patient data from the NTDB. Their clinical variables to stratify VTE risk and to determine prophylaxis requirements included the following: age, ISS, obesity (yes/no), ventilator use (yes/no), and lower extremity fracture (yes/no).

In the military setting, Hutchison et al., and Gillern et al., both reported bilateral lower extremity fracture as a significant risk factor for VTE development [4, 8]. Hutchison identified the highest risk for VTE to be multiple-limb amputations and above-knee amputations. Extremity, abdomen, and head AIS showed correlation on univariate analysis, but the relationship did not persist on multivariate analysis. Holley et al. analyzed a subset of patients from the Hutchison study and found blood transfusion predictive of VTE and receiving $>50 \%$ of prescribed LMWH prophylaxis doses as protective [10•].

Risk factors for VTE in civilian and military trauma consistently include specific injury patterns involving the lower extremities (e.g., long bone fractures and amputations.)

\section{Acute Peritraumatic Pulmonary Thrombus}

Recently, the dissociation of pulmonary embolism from deep venous thrombosis has been proposed by several authors [2, 11]. They suggest the etiology of pulmonary VTE to be primary thrombosis within the pulmonary vasculature itself, not venous embolism, especially when associated with primary chest trauma. Knudson hypothesized that local inflammation from chest trauma as a fourth risk factor to explain increasing rates of civilian PE without the presence of coincident DVT [2]. These events have been labeled as acute peritraumatic pulmonary thrombus (APPT).

Lundy et al. noted an APPT rate of $9.3 \%$ in reviewing initial CT scans of 708 consecutive combat-injured patients from July 1, 2011-June 30, 2012 in southern Afghanistan (Camp Bastion) [11]. APPT was associated with blast injuries, higher ISS, lower extremity DVT on CT scan, traumatic amputations, long bone fractures, tourniquet use, and transexamic acid (TXA) administration. Logistic regression identified traumatic amputation and DVT on CT scan (although only $23 \%$ had DVT present on CT scan) to be independently associated with APPT. Like Velmahos et al. [11] and Knudson et al. [2], Lundy et al. [12•] found widely disparate rates of DVT in patients with pulmonary thrombus, suggesting different mechanism for each entity.

The subsequent natural history of APPT has not been determined, but would likely be determined by location of thrombus and associated physiologic changes (e.g., presence or absence of right heart strain). In the Lundy study, $50 \%$ of APPT was 
not diagnosed at the time of initial CT interpretation; therefore, a cohort of patients who did not receive anticoagulation exists who could serve as a control group for future study. Some authors suggest that incidental, subsegmental PEs on CT imaging might not require therapeutic anticoagulation, and that may as well be the case with APPT.

\section{Air Travel and Venous Thromboembolism Risk}

The US military trauma care system makes the patient a mobile part of the system. Life-saving interventions are performed, and essential care is delivered at forward, deployed locations. Patients then evacuate across increasingly capable levels of care, culminating with arrival at facilities in the USA for definitive care and rehabilitation. Thus, from point of injury on the battlefield, treatment of combat casualties is a multi-step process frequently $3-5$ days of immobility and hours of flight on multiple airframes [13] [Fig. 1].

Few studies exist assessing VTE risk associated with surgery and flying. In 2014, Cooper et al. compared VTE rates of 220 postoperative patients who flew home in the immediate postoperative period (mean 2.9 days, mean flight time $2.3 \mathrm{~h}$ ) following total hip and total knee arthroplasty to 1245 similarly postoperative patients who did not fly [14]. This study did not find differences in VTE rates (OR 1.13 [0.33-3.95], $p=0.84$ ). In contrast, Gajic et al. found prolonged air travel before major surgery increased perioperative VTE rates ( $4.9 \%$ flight vs $0.15 \%$ no flight, $p<0.001$ ) and urged "more intensive" VTE prophylactic measures for these patients [15]. Current literature suggests $>4 \mathrm{~h}$ flight time as the threshold for increased VTE risk with contributory factors attributed to air travel including hypoxia, cramped sitting, low ambient humidity, and dehydration [16].

In 2001, the World Health Organization launched a comprehensive investigation to study the effects of air travel on VTE risk as a global health hazard. The WRIGHT Project, WHO Research into Global Hazards of Travel, consists of research studies designed to provide information on the following: (1) the frequency of venous thrombosis, (2) the magnitude of its association with air travel, and (3) the pathophysiology to develop prevention strategies for air travelers. The final report of phase I of the WRIGHT Project was released in 2007 [17]. Conclusions derived from the epidemiologic and pathophysiologic studies presented are concerning for military casualties undergoing aeromedical evacuation.

Phase I findings indicate that in air travelers who are not traumatically injured:

- VTE risk doubles after $q$ long-haul flight $>4 h$ duration) — tactical (in-theater) aeromedical evacuation flight times may approach a 4-h duration. All strategic (intra-theater) aeromedical evacuation flight times exceed $4 \mathrm{~h}$.

- VTE risk increases with total duration of travel and increases with multiple flights within a short period - casualties, especially those most severely injured, fly as many as three times within a few days to rapidly evacuate to the role 5 facilities in the USA for definitive care. Flight times from role 4 to role 5 military medical treatment facilities may exceed $10 \mathrm{~h}$.
Fig. 1 From point of injury on the battlefield, treatment of combat casualties is a multi-step process frequently $3-5$ days of immobility and hours of flight on multiple airframes
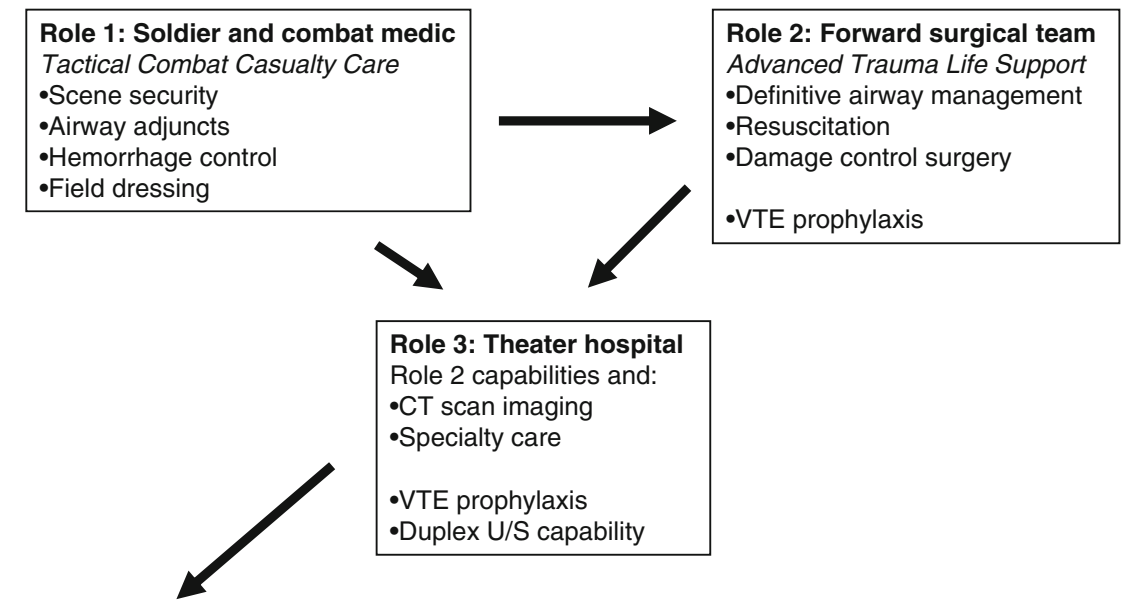

Role 4: Strategic evacuation medical center - Located outside of the U.S.

-Role 3 capabilities and:

- Limited short-term rehabilitation resources

-VTE prophylaxis

-Duplex U/S capability

-IVC filter placement capability (inconsistent) 
- VTE risk is highest in the first week after travel and remains elevated for 2 months after travel - pharmaceutical VTE prophylaxis during this period may be contraindicated due to hemorrhage risk from traumatic injuries and surgical interventions.

Thus, in addition to trauma-related risk factors for VTE complications, military casualties also endure added risks associated with air travel across the global combat casualty care continuum originating at the combat theaters to definitive care destinations in the USA.

\section{Early Venous Thromboembolism Incidence in Severely Injured Combat Trauma Patients (Role 4 Facility: Landstuhl, Germany)}

After battlefield injury and immediate treatment at deployed medical treatment facilities in the combat zone (role 2 and role 3 ), nearly all injured service members are evacuated to a role 4 facility. For combat casualties injured in Iraq or Afghanistan, that role 4 facility is Landstuhl Regional Medical Center (LRMC) in Germany. Recognizing the extremely high risk for VTE complications for the combat-injured casualties, the LRMC Trauma Program instituted a policy of universal DVT screening with bilateral lower extremity Duplex ultrasound for trauma admissions to the critical care unit in 2010. It was believed that earlier DVT detection would allow earlier treatment, thereby decreasing the incidence of subsequent $\mathrm{PE}$ events. After 12 months, a performance improvement project was performed to assess the effectiveness of universal DVT screening policy [18].

All trauma patients admitted to the critical care unit at LRMC from August 1, 2009 to July 31, 2011 were retrospectively evaluated. Most patients arrived within $72 \mathrm{~h}$ of injury after aeromedical evacuation from role 3 facilities in Iraq and Afghanistan. Patients in cohort 1 (C1, August 1, 2009July $31,2010, n=497$ ) selectively underwent lower extremity duplex ultrasound imaging based upon physician discretion. Patients in cohort 2 (C2, August 1, 2010-July 31, 2011, $n=565$ ) underwent universal DVT screening. PEs were diagnosed by computed tomography imaging, either specifically obtained for clinical suspicion of PE or incidentally discovered on imaging for other indications. In casualties with PEs, their preceding lower extremity duplex ultrasound examinations were reviewed and classified as either "complete" or "limited." Limited screenings were generally related to lower extremity wound dressings or orthopedic hardware preventing complete interrogation of the underlying deep veins.

Injury severity scores were equivalent between the two cohorts $(\mathrm{C} 1=21.9, \mathrm{C} 2=21.9, p=\mathrm{NS})$. Thirty-two DVT and $7 \mathrm{PE}$ were found in $\mathrm{C} 1$ and $80 \mathrm{DVT}$ and $25 \mathrm{PE}$ in $\mathrm{C} 2$ (DVT incidence $6.44 \%$ vs. $14.15 \%, p<0.0001$ and PE incidence
$1.40 \%$ vs. $4.42 \%, p=0.0072$ ). In patients with PE, 2 of the 7 patients in $\mathrm{C} 1$ and 15 of the 25 patients in C2 had DVT examinations classified as limited ( $29 \%$ vs. $60 \%, p=\mathrm{NS}$ ).

This performance improvement project demonstrated that universal DVT screening diagnosed significantly more casualties with DVT than selective imaging. Surprisingly, PE incidence increased with universal DVT screening potentially related to increased utilization of $\mathrm{CT}$ chest imaging for a variety of indications leading to increased detection of incidental PE or APPT. The LRMC Trauma Program continued universal DVT screening for trauma admissions to the critical care unit following this performance improvement analysis.

\section{Prevention of Deep Venous Thrombosis}

The Joint Trauma System (JTS) is a Department of Defense (DoD) organization created to ensure optimal management of the combat injured across the global care continuum. The JTS maintains the DoDTR and coordinates clinical practice guidelines (CPG) based upon objective data from the registry, published medical literature, and consensus expert opinion. The JTS first released a CPG for "Prevention of deep venous thrombosis" in December 2004 which has undergone regular review and revision to ensure it reflects current, best practice [19]. For trauma patients, the JTS currently recommends low-molecular weight heparin (LMWH) $30 \mathrm{mg}$ subcutaneously twice daily for VTE prophylaxis with strong consideration to add sequential compression devices (SCDs) as well if both feasible and available. In trauma patients with prohibitive risks of bleeding or with ongoing coagulopathy, SCDs are recommended until pharmacologic prophylaxis can be safely commenced. "It is possible that certain patients will still be receiving blood product therapy to correct coagulopathy when they enter the [evacuation] chain... It is recommended to begin [pharmacologic] DVT prophylaxis therapy as soon as coagulopathy is corrected in patient not otherwise at risk of bleeding." For brain and spinal injuries, full-dose pharmacologic prophylaxis was initiated after $48 \mathrm{~h}$ of clinical and radiographic stability. Pharmacologic prophylaxis is also not routinely held prior to operative interventions except those with the highest risk of bleeding complications such as brain and spinal procedures.

While LMWH is generally the preferred prophylactic agent for trauma patients, the optimal dosing regimen remains controversial [20, 21]. Recent studies show that VTE rates may be directly correlated with anti-factor Xa activity levels, so some VTE prophylaxis protocols incorporate activity level monitoring to ensure adequacy [22]. Activity level monitoring is generally not available prior to role 3 care and consensus does not exist for the monitoring requirement. 
The JTS CPG also provides guidance for the placement of inferior vena caval filters (IVCF). It notes that:

when IVCF are placed they are done so to prevent FATAL pulmonary emboli as PE's can still occur. IVCF have no benefit in the prevention of DVT and may be associated with development of IVC and deep venous thrombosis... The vast majority of IVCF placed in the combat theater are retrievable inferior vena cava filters. Retrievable IVCF are preferred to avoid some of the long term complications of filter placement and in recognition of the fact that many patients only need this form of VTE prophylaxis for a defined period of time early after injury.

Standard indications for IVCF placement are used for military casualties with additional consideration for placement in very high-risk patients who cannot receive prophylactic anticoagulation because of increased bleeding risk especially at times when SCDs are also not available for use. Prior to April 2009, no SCD system was approved for use during aeromedical evacuation until the Kendall-Covidien 9525 SCD Express Compression System passed airworthiness testing.

IVCF placement is not consistently available prior to role 5 military medical treatment facilities. When placed, IVCF should be retrievable and the brand, model, MRI compatibility, and specific placement location should be populated in the DoDTR. Typically, IVCF retrieval will occur at Role 5 facilities and should occur as soon as contraindications to pharmacologic prophylaxis no longer exist or there is no longer need for VTE prophylaxis. In 2010, the US Food and Drug Administration posted a communication expressing concern that retrievable IVCFs, intended for short-term placement, are not always removed once a patient's PE risk subsides [23]. The FDA listed long-term risks associated with IVC filters including lower limb DVT, filter fracture, filter migration, filter embolization, and IVC perforation. Beyond 3 months of implantation, IVCF removal may become technically prohibitive and so these longterm risks must be carefully considered.

Inferior vena cava interruption for VTE management in combat trauma has led to variable results. In 2009, Johnson et al. published their early experience with 72 IVCFs placements from July 2003-June 2007 at the Walter Reed Army Medical Center [24]. In this series, 23 insertions were prophylactic and 49 were 'therapeutic' following a VTE event when post-event therapeutic anticoagulation was contraindicated. There were no untoward events associated with filter placement, but only 13 (18\%) filters were successfully removed. Eleven IVCF patients (15\%) were lost to follow-up, 45 (63\%) kept their filters for ongoing clinical indications and one died. IVCF retrieval was attempted in 15 IVCF patients (21\%), and 13 attempts were successful. In comparison to contemporary civilian literature at the time, the authors reported a significantly higher patient follow-up, but a similarly low overall retrieval rate due to filters remaining clinically indicated rather than the patient being lost to follow-up.

Lucas et al. also reported results of a dedicated military IVCF registry developed in 2007 [25]. The study compared patient follow-up and filter retrieval rates pre- and postregistry initiation at NNMC-Bethesda. The study included 113 patients (20 in the control group and 93 in the registry). Trauma nurse coordinators maintained routine communication with IVCF patients. This process led to a decrease in loss to follow-up from $65 \%$ to $5 \%(p<0.001)$ and a significant increase in retrieval rates from $30 \%$ to $60 \%(p=0.02)$. The registry and communication process remains active today at the Walter Reed National Military Medical Center (Bethesda, Maryland).

Another management strategy for prevention of PE, but not prevention of DVT, in high-risk patients described in the JTS CPG is serial duplex ultrasound imaging. Earlier DVT detection may facilitate treatment, therapeutic anticoagulation, or IVCF placement, to decrease the incidence of subsequent PE. In high-risk patients with short-term contraindications to pharmacologic prophylaxis, serial duplex ultrasound imaging was sometimes performed twice weekly until pharmacologic prophylaxis could be initiated. If a DVT was detected, IVCF placement was performed.

\section{Conclusion}

VTE complications impact the care of combat casualties who are an extremely high-risk cohort due to their patterns of injury, injury severity, and the requirement for aeromedical evacuation. Even so, the overall civilian versus military VTE rates comparing population-based retrospective databases, NTDB vs DoDTR, differed only in the rates of PE (DVT: $1.06 \%$ NTDB vs $1.0 \%$ DoDTR and PE: $0.42 \%$ NTDB vs $0.8 \%$ DoDTR). Reviews of selected high-risk cohorts such as critical care admissions and severe extremity injuries revealed VTE rates as high as $20 \%$ despite VTE prophylaxis measures. Some cases of PE may represent APPT, be present on initial trauma admission, and be non-preventable. The DoD's Joint Trauma System's mission remains to guide optimal management of military trauma care to include the prevention, detection, and management of VTE disease based upon DoDTR data and monitoring of the published civilian trauma experience.

\section{Compliance with Ethical Standards}

Conflict of Interest The authors declare that they have no conflicts of interest.

Human and Animal Rights and Informed Consent This article does not contain any studies with human or animal subjects performed by any of the authors. 


\section{References}

Papers of particular interest, published recently, have been highlighted as:

- Of importance

1. Geerts WH, Code KI, Jay RM, Chen E, Szalai JP. A prospective study of venous thromboembolism after major trauma. N Engl $\mathrm{J}$ Med. 1994;331(24):1601-6.

2. Knudson MM, Gomez D, Haas B, Cohen MJ, Nathens AB. Three thousand seven hundred thirty-eight posttraumatic pulmonary emboli: a new look at an old disease. Ann Surg. 2011;254(4):625-32.

3. Brakenridge SC, Henley SS, Kashner TM, Golden RM, Paik DH, Phelan HA, et al. Inflammation and the host response to injury investigators. Comparing clinical predictors of deep venous thrombosis versus pulmonary embolus after severe injury: a new paradigm for posttraumatic venous thromboembolism? J Trauma Acute Care Surg. 2013;74(5):1231-7. Authors present the theory that after severe blunt injury, DVT and PE represent independent thrombotic entities rather than different stages of a single pathophysiologic process.

4. Hutchison TN, Krueger CA, Berry JS, Aden JK, Cohn SM, White CE. Venous thromboembolism during combat operations: a 10-y review. J Surg Res. 2014;187(2):625-30.

5. Caruso JD, Elster EA, Rodriguez CJ. Epidural placement does not result in an increased incidence of venous thromboembolism in combat-wounded patients. J Trauma Acute Care Surg. 2014;77

(1):61-6. This paper found no difference in VTE incidence between enoxaparin $40 \mathrm{mg}$ daily in patients with patientcontrolled epidural anesthesia versus $30 \mathrm{mg}$ twice daily for VTE prophylaxis in combat-wounded patients. However, 55/ 484 patients developed VTE during hospitalization despite prophylaxis.

6. Arroyo W, Nelson KJ, Belmont Jr PJ, Bader JO, Schoenfeld AJ. Pelvic trauma: what are the predictors of mortality and cardiac, venous thrombo-embolic and infectious complications following injury? Injury. 2013;44(12):1745-9.

7. El-Daly I, Reidy J, Culpan P, Bates P. Thromboprophylaxis in patients with pelvic and acetabular fractures: a short review and recommendations. Injury. 2013;44(12):1710-20.

8. Gillern SM, Sheppard FR, Evans KN, Graybill JC, Gage FA, Forsberg JA, et al. Incidence of pulmonary embolus in combat casualties with extremity amputations and fractures. J Trauma. 2011;71(3):607-12.

9. Rogers FB, Shackford SR, Horst MA, Miller JA, Wu D, Bradburn E, et al. Determining venous thromboembolic risk assessment for patients with trauma: the Trauma Embolic Scoring System. J Trauma Acute Care Surg. 2012;73(2):511-5.

10. Holley AB, Petteys S, Mitchell JD, Holley PR, Collen JF. Thromboprophylaxis and VTE rates in soldiers wounded in operation enduring freedom and operation Iraqi freedom. Chest. 2013;144 (3):966-73. Review of 506 combat casualties admitted to Walter Reed with daily notation of their VTE prophylaxis regimen amongst other clinical data. Enoxaparin $30 \mathrm{mg}$ twice daily decreased VTE events compared to once daily dosing or prophylaxis with unfractionated heparin.

11. Velmahos GC, Spaniolas K, Tabbara M, Abujudeh HH, de Moya M, Gervasini A, et al. Pulmonary embolism and deep venous thrombosis in trauma: are they related? Arch Surg. 2009;144(10): 928-32.

12. Lundy JB, Oh JS, Chung KK, Ritter JL, Gibb I, Nordmann GR, et al. Frequency and relevance of acute peritraumatic pulmonary thrombus diagnosed by computed tomographic imaging in combat casualties. J Trauma Acute Care Surg. 2013;75(2 Suppl 2):S21520. APPT was found in $66(9.3 \%)$ of 708 consecutive trauma admissions, which received a $\mathrm{CT}$ chest with intravenous contrast as part of their initial trauma evaluation.

13. Fang R, Dorlac GR, Allan PF, Dorlac WC. Intercontinental aeromedical evacuation of traumatic brain injuries during Operations Iraqi Freedom and Enduring Freedom. Neurosurg Focus. 2010;28 (5):E11.

14. Cooper HJ, Sanders SA, Berger RA. Risk of symptomatic venous thromboembolism associated with flying in the early postoperative period following elective total hip and knee arthroplasty. J Arthroplasty. 2014;29(6):1119-22.

15. Gajic O, Warner DO, Decker PA, Rana R, Bourke DL, Sprung J. Long-haul air travel before major surgery: a prescription for thromboembolism? Mayo Clin Proc. 2005;80(6):728-31.

16. Schobersberger W, Fries D, Mittermayr M, Innerhofer P, Sumann G, Schobersberger B, et al. Changes of biochemical markers and functional tests for clot formation during long-haul flights. Thromb Res. 2002;108(1):19-24.

17. World Health Organization (Geneva, Switzerland). WHO research into global hazards of travel (WRIGHT) Project: Final report of phase I. Geneva, Switzerland): WHO Press; 2007. 24 p.

18. Stamp TW, Schell E, Martin K, Fang R, Zonies DH, Oh JS. Thromboembolic events in combat trauma patients: impact of universal screening. Poster presented at: Transformational pathways to global healthcare. AMSUS The Society of the Federal Healthcare Agencies 117th Annual Meeting; 2011 November 6-9; San Antonio, Texas.

19. United States Army Institute of Surgical Research. JTS \& CCAT Clinical Practice Guidelines. Available at: http://usaisr.amedd.army. mil/cpgs.html. Accessed February 9, 2016.

20. Riha GM, Van PY, Differding JA, Schreiber MA. Oregon Health \& Science University Trauma Research Group. Incidence of deep vein thrombosis is increased with $30 \mathrm{mg}$ twice daily dosing of enoxaparin compared with $40 \mathrm{mg}$ daily. Am J Surg. 2012;203(5): 598-602.

21. Gritsiouk Y, Hegsted DA, Schlesinger P, Gardiner SK, Gubler KD. A retrospective analysis of the effectiveness of low molecular weight heparin for venous thromboembolism prophylaxis in trauma patients. Am J Surg. 2014;207(5):648-51. discussion 651-2.

22. Malinoski D, Jafari F, Ewing T, Ardary C, Conniff H, Baje M, et al. Standard prophylactic enoxaparin dosing leads to inadequate antiXa levels and increased deep venous thrombosis rates in critically ill trauma and surgical patients. J Trauma. 2010;68(4):874-80.

23. U.S. Food and Drug Administration. Removing Retrievable Inferior Vena Cava Filters: FDA Safety Communication (Date of Initial Communication: August 9, 2010, Date Updated: May 6, 2014). Available at: http://www.fda.gov/MedicalDevices/Safety/ AlertsandNotices/ucm396377.htm. Accessed February 9, 2016.

24. Johnson 3rd ON, Gillespie DL, Aidinian G, White PW, Adams E, Fox CJ. The use of retrievable inferior vena cava filters in severely injured military trauma patients. J Vasc Surg. 2009;49(2):410-6. discussion 416.

25. Lucas DJ, Dunne JR, Rodriguez CJ, Curry KM, Elster E, Vicente $\mathrm{D}$, et al. Dedicated tracking of patients with retrievable inferior vena cava filters improves retrieval rates. Am Surg. 2012;78(8):870-4. 